\title{
Bilateral Spike-Triggered Average Effects in Arm and Shoulder Muscles from the Monkey Pontomedullary Reticular Formation
}

\author{
Adam G. Davidson, ${ }^{1,3}$ Marc H. Schieber, ${ }^{4}$ and John A. Buford ${ }^{2}$ \\ ${ }^{1}$ Neuroscience Graduate Studies Program and ${ }^{2}$ School of Allied Medical Professions, The Ohio State University, Columbus, Ohio 43210, and ${ }^{3}$ Department of \\ Neurobiology and Anatomy and ${ }^{4}$ Departments of Neurology, Brain and Cognitive Science, Physical Medicine, and Rehabilitation, The Center for Visual \\ Science and the Brain Injury Rehabilitation Program at St. Mary's Hospital, University of Rochester School of Medicine and Dentistry, Rochester, New York \\ 14642
}

Pontomedullary reticular formation (PMRF) neurons (309) were recorded simultaneously with electromyographic activity from arm and shoulder muscles in four monkeys performing arm-reaching tasks. Spike-triggered averages (SpikeTAs) were compiled for 292 neurons (3836 neuron-muscle pairs). Fourteen PMRF neurons located in a region ventral to the abducens nucleus produced 42 significant SpikeTA effects in arm and shoulder muscles. Of these 14 PMRF neurons, nine produced SpikeTA effects bilaterally. Overall, PMRF neurons facilitated ipsilateral flexors and contralateral extensors, while suppressing ipsilateral extensors and contralateral flexors. Spike- and stimulus-triggered averaging effects obtained from the same recording site were similar. These findings indicate that single PMRF neurons can directly influence movements of both upper limbs.

Key words: upper limb; brainstem; motor control; reticular; EMG; primate

\section{Introduction}

Reticulospinal neurons have axons that originate in the pontomedullary reticular formation (PMRF) and descend via the medial and lateral reticulospinal tracts, targeting axial and limb motoneurons bilaterally, either through interneurons or via monosynaptic connections (Peterson, 1979; Jankowska et al., 2003). Cat reticulospinal neurons contribute to the control of locomotion and of forelimb reaching movements (Schepens and Drew, 2006). Previous evidence indicates that monkey PMRF neurons also show activity related to preparation and performance of reaching (Buford and Davidson, 2004). In the monkey PMRF, stimulus-triggered averages (StimulusTA) of rectified electromyographic (EMG) activity revealed output effects in forearm, arm, and shoulder muscles bilaterally (Davidson and Buford, 2004, 2006). Here, we used spike-triggered averaging to examine the extent to which monkey PMRF neurons contribute directly to control of upper limb muscles during reaching.

\section{Materials and Methods}

Monkeys and tasks. Single-unit activity was recorded in the PMRF simultaneously with forelimb EMG activity in four Macaca fascicularis monkeys performing instructed-delay arm reaching tasks. Procedures com-

\footnotetext{
Received Jan. 5, 2007; revised June 19, 2007; accepted June 21, 2007.

This work was supported by National Institute of Neurological Disorders and Stroke Grants R01 NS037822 (J.A.B.) and R01/R37-NS27686 (M.H.S.). We thank Stephanie Moran for technical assistance.

Correspondence should be addressed to Dr. Adam G. Davidson, University of Rochester Medical Center, Department of Neurobiology and Anatomy, 601 Elmwood Avenue, Box 603, Rochester, NY 14642. E-mail: adam_davidson@urmc.rochester.edu.

DOl:10.1523/JNEUROSCI.0040-07.2007

Copyright $\odot 2007$ Society for Neuroscience $\quad$ 0270-6474/07/278053-06\$15.00/0
}

plied with the National Institutes of Health Guide for the Care and Use of Laboratory Animals and the institutionally approved animal care protocol. Monkeys $\mathrm{C}$ and $\mathrm{D}$ performed a planar, center-out reaching task with the right arm; the task-related activity of their PMRF neurons has been described previously (Buford and Davidson, 2004). Monkeys I and G reached with either arm to targets presented on a touch screen monitor, and retrieved pellets from a food well located below the touch screen (Davidson and Buford, 2006). The task-related activity of their PMRF neurons will be presented in a forthcoming manuscript.

Neural and EMG recording procedures. Surgical procedures for chamber and EMG implantation, recording procedures, and the anatomical reconstructions were presented previously (Davidson and Buford, 2004, 2006). Polyimide-insulated tungsten microelectrodes (Frederick Haer, Bowdoinham, ME) were used for extracellular neuron recordings at sites within the nucleus reticularis pontis caudalis (NRPc) and the rostral part of nucleus reticularis gigantocellularis (NRGc) in the PMRF. EMG electrodes were pairs of multistranded, Teflon-coated stainless-steel wires (Cooner Wire, Chatsworth, CA). In monkeys I and G, EMG electrodes were implanted chronically in the following muscles of both the right and left upper extremity: biceps, long head (Bic); triceps lateral (TrLa) or long (TrLo) head; anterior deltoid (ADlt); posterior deltoid (PDlt); pectoralis major (PMj); latissimus dorsi (Lat); middle trapezius (MTr); upper trapezius (UTr); flexor carpi radialis (FCR); extensor carpi ulnaris; and brachioradialis (Brac). In monkey C, EMG electrodes were implanted in Bic, PDlt, TrLo, PMj, Lat, and UTr on the right, and in MTr and UTr on the left. In monkey D, EMG electrodes were implanted in Bic, ADlt, PDlt, TrLo, MTr, and UTr on the right side, and in UTr on the left. EMG data were excluded from analysis if postmortem examination indicated the EMG electrodes had dislodged from the muscle. Evidence of cross talk between TrLa and TrLo EMG implants was found occasionally. Consequently, one spike-triggered average (SpikeTA) effect was selected for analysis when TrLa and TrLo both showed SpikeTA effects within the 
same limb. For analysis, the SpikeTA effects accepted from either TrLa or TrLo were combined as triceps (Tri). In total, EMG data from 40 muscles were analyzed with SpikeTA (monkey I, 15; G, $13 ; C, 7 ; D, 5)$. We refer to muscles as ipsilateral (i) or contralateral (c) to the recording site. Neural, EMG, and behavioral data were recorded with Spike2 software and a Power 1401-PC interface (CED, Cambridge, England). Single-pulse electrical stimulation for StimulusTA was applied at most neuron recording sites, either after a recording or during electrode withdrawal (Davidson and Buford, 2004, 2006). Stimulation for StimulusTA was biphasic pulses $(0.2 \mathrm{~ms}$ per phase) of 25-40 $\mu \mathrm{A}$ (mean, $30.4 \mu \mathrm{A}$ ) applied at $13.3 \mathrm{~Hz}$ (monkeys $\mathrm{C}$ and $\mathrm{D}$ ) or $10 \mathrm{~Hz}$ (monkeys I and $\mathrm{G})$.

Spike-triggered averaging and analysis. SpikeTAs were compiled off-line for neuron-muscle pairs with at least 4000 recorded action potentials. EMG was rectified and averaged over an 80 $\mathrm{ms}$ interval beginning $30 \mathrm{~ms}$ before and ending $50 \mathrm{~ms}$ after the spike. Spikes were included from all task periods, including movements of either arm. Spikes were accepted as triggers if they occurred when ongoing EMG activity was present in the channel being averaged [i.e., if during the $80 \mathrm{~ms}$ interval around the spike, the root mean squared (rms) EMG signal was $>110 \%$ of the rms noise level for that channel]. Excluding spikes that occur without EMG activity eliminates sweeps containing only amplifier noise and provides a SpikeTA that more accurately reflects the effect of the neuron on the EMG activity of the muscle (McKiernan et al., 1998; Schieber and Rivlis, 2005). The resulting averages were smoothed with a five-point flat filter.

Significant SpikeTA effects were identified with multifragment statistical analysis (Poliakov and Schieber, 1998). We tested for significant peaks or troughs within a $12 \mathrm{~ms}$ test window, from 3 to $15 \mathrm{~ms}$ after the trigger, because StimulusTA from the PMRF produced effects with onsets between 3.5 and $15 \mathrm{~ms}$ (Davidson and Buford, 2004, 2006), and we allowed $0.5 \mathrm{~ms}$ for stimulus utilization time. Only peaks or troughs occurring in this 3-15 $\mathrm{ms}$ window were analyzed in the present report. If a neuron produced at least one SpikeTA effect that remained statistically significant at the $p<$ 0.05 level after Bonferroni correction for testing multiple neuron-muscle pairs, then we accept that neuron as producing effects, and any SpikeTA effect produced by that neuron with an uncorrected $p<0.05$ was accepted as a significant effect.

The temporal and amplitude characteristics of SpikeTA effects were determined relative to the mean value of the average EMG in a $20 \mathrm{~ms}$ baseline period ( -30 to $-10 \mathrm{~ms}$ from the trigger). Onset and offset latencies and peak width at half maximum (PWHM) were measured to quantify the temporal characteristics; peak amplitude, peak percentage change (PPC), and mean percentage change (MPC) were measured to quantify the amplitude characteristics. Onset and offset latencies were defined as the times when the averaged EMG crossed a level two SDs from the baseline mean before and after the peak (or trough). PWHM was measured as the width of a SpikeTA effect at half the peak (or trough) amplitude, where peak amplitude was measured as the difference between the average EMG baseline mean and the maximum (or minimum) value during the SpikeTA effect. PPC was the difference between the peak amplitude and EMG baseline mean expressed as a percentage of the baseline mean; MPC was the difference between the mean of SpikeTA effect from onset to offset and the EMG baseline expressed as a percentage of the baseline mean. Onset latency, PWHM, PPC, and MPC were compared for response type (facilitation vs suppression) and laterality (ipsilateral vs contralateral) with ANOVA and post hoc $t$ tests. The signif-
B

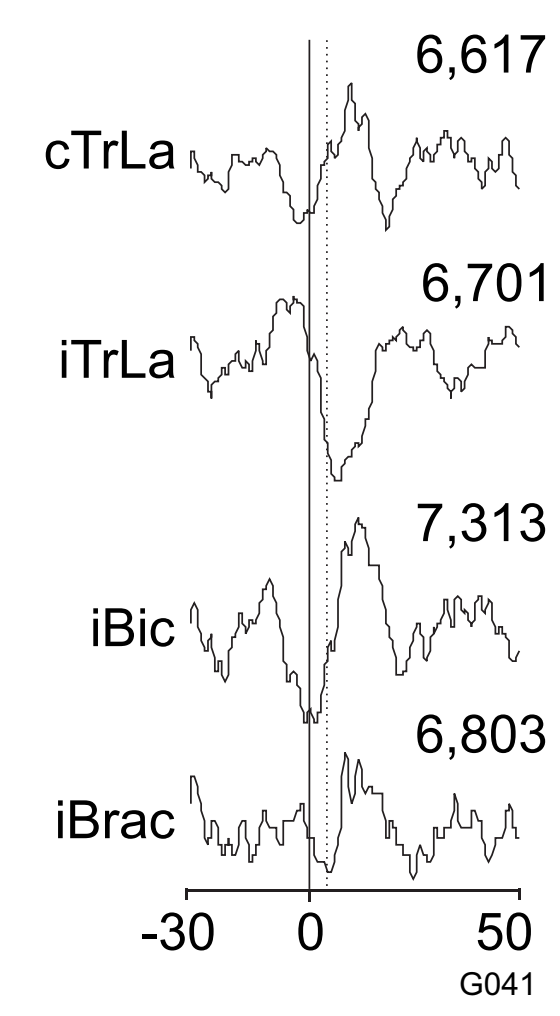

$114 \mathrm{H}$

\section{1,907}

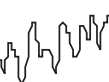

21,880

18,890 19,882

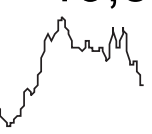

50

(1)
20,775

(1)

Figure 1. PMRF neuron spike-triggered averages of EMG from arm and shoulder muscles. $A, B$, SpikeTAs from monkey I (I14H; A) and monkey $G(G 041 ; \boldsymbol{B})$. For all figures, SpikeTAs are compiled over $80 \mathrm{~ms}(-30$ to $+50 \mathrm{~ms})$. The solid vertical lines indicate above each neuron-muscle pair.

icance level was set to $p<0.05$ for all statistical comparisons among SpikeTA effect measures.

SpikeTA effects with appropriate onset latencies and narrow PWHMs (typically referred to as postspike effects) reflect relatively direct synaptic input from the trigger neuron to the motoneuron pool producing the recorded EMG. Other SpikeTA effects with early onset latencies and/or wide PWHMs (referred to as synchrony effects) reflect additional synaptic inputs to the motoneuron pool synchronized with the spikes of the trigger neuron (Schieber and Rivlis, 2005). All significant SpikeTA effects were included in the present analysis, regardless of their onset latency and PWHM.

\section{Results}

Three hundred and nine PMRF neurons were recorded in four monkeys (27 in monkey C, 30 in D, 186 in I, and 66 in G). After 17 neurons were excluded for having $<4000$ recorded spikes, 292 neurons (3836 neuron-muscle pairs) were analyzed with SpikeTA. Overall, 14 neurons recorded from the four monkeys (two in C, two in D, six in I, four in G) produced 42 SpikeTA effects. Figure $1 A$ illustrates representative SpikeTA effects from one neuron in monkey I (Fig. $1 A ; \mathrm{I} 14 \mathrm{H}$ ) and one neuron in monkey G (Fig. $1 B$; G041). Although in these two examples facilitative SpikeTA effects were more prevalent than suppressive SpikeTA effects, comparable numbers of facilitative (23) and suppressive (19) SpikeTA effects were found overall. In these two examples, SpikeTA effects were produced in 33\% (5 of 15) (Fig. 1A) and $31 \%$ (4 of 13) (Fig. 1B) of the simultaneously recorded muscles. Overall, 11 of the 14 neurons produced SpikeTA effects in more than one muscle; three neurons produced a SpikeTA effect in one muscle. 
A

\section{SpikeTA}

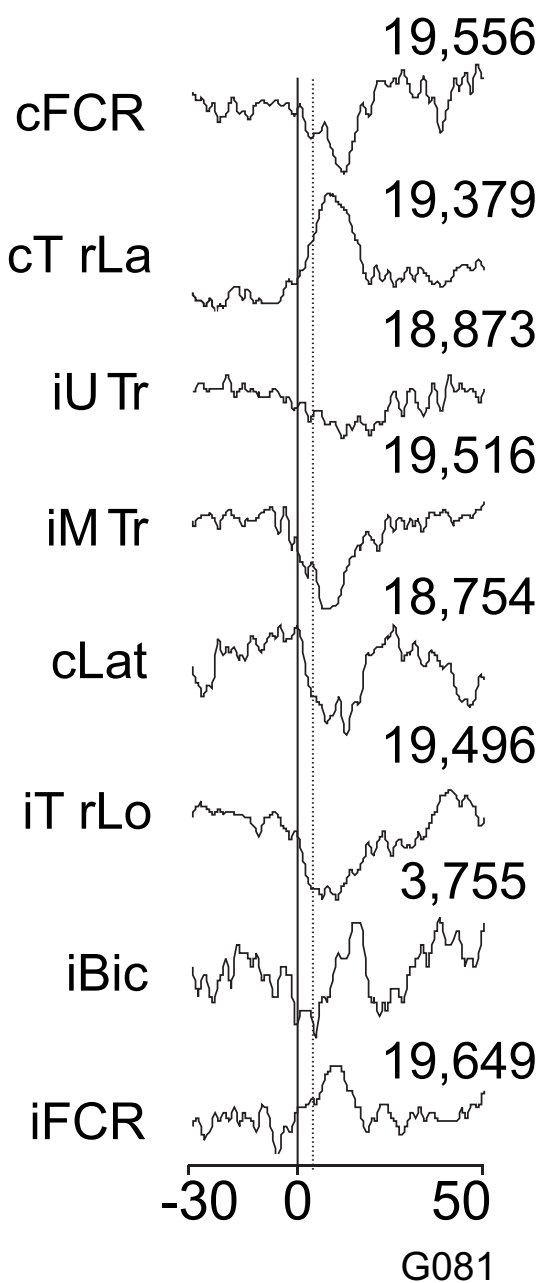

\section{StimulusTA}

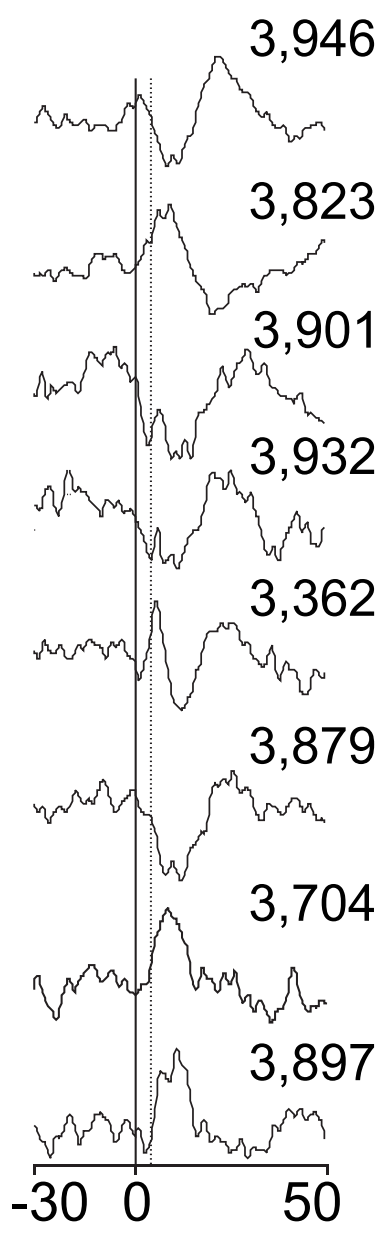

B

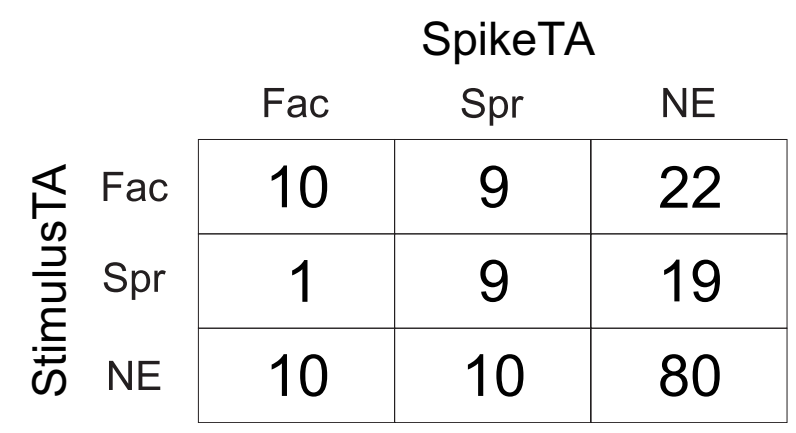

Figure 2. Comparison between spike- and stimulus-triggered averages obtained at the same PMRF site. $A$, SpikeTA compiled from neuron G081 (left) and the StimulusTA (right) compiled from stimulation applied at the same site immediately after the neuron recording (30 $\mu$ A). B, Overall counts for suppressive (Spr) and facilitative (Fac) SpikeTA and StimulusTA effects, or no effect (NE) for sites at which PMRF neurons produced SpikeTA effects and stimulation was applied for StimulusTA.

(Buford and Davidson, 2004), we were able to compare reach related discharge patterns and SpikeTA effects during reaches in four different directions. Each of these four neurons showed similar discharge patterns, and similar SpikeTA effects, during reaches to each of the four targets. In this limited sample, we thus observed no related variation in the SpikeTA effects and the movement related discharge of individual PMRF neurons.

\section{Single PMRF neurons produced SpikeTA effects bilaterally}

Of the 14 PMRF neurons that produced SpikeTA effects, nine (64\%) produced SpikeTA effects bilaterally, as illustrated for the two neurons whose SpikeTA effects are shown in Figure 1, $A$ and $B$. Although more ipsilateral effects $(n=24)$ than contralateral effects $(n=18)$ were found, this difference could be attributed to sampling ipsilateral muscles more frequently. The proportion of neuron-muscle pairs with versus without a SpikeTA effect did not differ significantly for ipsilateral (24 of 312 ) versus contralateral (18 of 234) muscles $\left(\chi^{2}\right.$ test; $\left.p>0.05\right)$.

\section{Reciprocal patterns of SpikeTA effects from PMRF neurons}

Of the 42 SpikeTA effects, 23 (55\%) were facilitative and 19 (45\%) were suppressive. The proportion of facilitative versus suppressive effects did not differ in the ipsilateral (15 facilitative, 13 suppressive effects) versus contralateral ( 8 facilitative, 6 suppressive) upper limb ( $p>0.05$, Fisher's exact test). Rather than being randomly distributed, however, these effects showed two patterns of reciprocal organization. First, within a limb, a given neuron tended to produce opposite effects in antagonistic muscles. One neuron, for example, suppressed $\mathrm{cPMj}$, a shoulder flexor, while facilitating cPDlt, a shoulder extensor (Fig. $1 A)$; another neuron suppressed iTrLa, an elbow extensor, while facilitating iBic, an elbow flexor (Fig. $1 B$ ). Of six instances in which a PMRF neuron produced SpikeTA effects in both members of an antagonist muscle pair acting about the same joint, the two SpikeTA effects were always reciprocal. In three instances, a PMRF neuron produced SpikeTA effects in a flexor and an extensor at separate joints, all of which were reciprocal.

Second, PMRF neurons tended to produce reciprocal SpikeTA effects in a given

On average, these 14 PMRF neurons produced SpikeTA effects in $28.5 \%$ of the simultaneously recorded muscles.

For the four neurons from monkeys C and D included in the population described previously during the planar reaching task muscle on the ipsilateral versus contralateral side. The neuron with SpikeTA effects illustrated in Figure $1 B$, for example, facilitated cTrLa while suppressing iTrLa. In six instances, a single PMRF neuron produced SpikeTA effects in a given muscle bilat- 
erally, and each of these six SpikeTA effect pairs were reciprocal. Single PMRF neurons thus could provide coordinated, reciprocal output to homologous muscles in the two arms.

These two patterns of reciprocal SpikeTA effects were evident as well in the overall population. The seven SpikeTA effects in ipsilateral flexor muscles (iADlt, iBic, iBrac, iFCR) were all facilitative, whereas in ipsilateral extensor muscles (iPDlt, iTrLo), 10 of 12 (83\%) SpikeTA effects were suppressive. In the contralateral extensors (cPDlt, cTrLa), all six effects were facilitative, whereas the two effects in the contralateral flexors (cPMj, cFCR) were suppressive. This association of ipsilateral flexors with facilitative effects versus ipsilateral extensors with suppressive effects was significant, as was the association of contralateral flexors with suppression versus contralateral extensors with facilitation ( $p<0.05$, Fisher's exact tests). Similarly, the association of ipsilateral flexors with facilitation versus contralateral flexors with suppression was significant, as was the association of ipsilateral extensors with suppression versus contralateral extensors with facilitation $(p<0.05$, Fisher's exact tests). Overall, PMRF neurons that produced SpikeTA effects supported a widespread, coordinated response in the two upper extremities, consisting of flexion in the ipsilateral, and extension in the contralateral upper extremity.

\section{Spike and stimulus-triggered averaging in the PMRF reveal similar motor outputs}

Single-pulse microstimulation was applied at the recording sites of 13 of the 14 PMRF neurons that produced SpikeTA effects (Davidson and Buford, 2004, 2006). Figure 2 illustrates the SpikeTA effects of one PMRF neuron and the StimulusTA effects obtained at the same site. In this example, SpikeTA effects were produced both ipsilaterally and contralaterally in forearm, arm, and shoulder muscles. All but one of these SpikeTA effects (cLat) was matched by a StimulusTA effect of the same sign (facilitation vs suppression). Of the 20 EMG recordings that had both a SpikeTA effect from a PMRF neuron and a StimulusTA effect from microstimulation at the site of that neuron, 10 showed facilitation in both the SpikeTA and stimulus-triggered average, nine showed suppression in both, and one showed facilitation in the SpikeTA, but suppression in the stimulus-triggered average. The overall matching of SpikeTA and stimulus-triggered average effects was significant $(p<0.001$, Fisher's exact test). Instances in which no effect was produced by SpikeTA, by StimulusTA, or by either were also counted for all 161 neuron-muscle pairs available from these 13 PMRF neurons. These counts are shown in Figure $2 B$. The matching of facilitation, suppression, or no effect produced by SpikeTA and StimulusTA at the same site also was significant $\left(p<0.0001, \chi^{2}\right.$ test $)$. The effects produced by stimulus-triggered averaging, thus, were similar to the SpikeTA effects of PMRF neurons recorded at the same site.

\section{Temporal and amplitude characteristics of PMRF neuron SpikeTA effects}

Figure 3 shows a scatter plot of onset latency versus PWHM for each of the 42 significant SpikeTA effects. Similar to populations of SpikeTA effects of M1 neurons (Schieber and Rivlis, 2005), later onset was correlated with narrower PWHM for SpikeTA effects from PMRF neurons. Also like M1 SpikeTA effects, onset latency and PWHM were distributed continuously, with little evidence of bimodal distributions that would suggest distinct categories of pure postspike effects versus effects reflecting synchronized input from additional neurons. Nevertheless, for purposes of exposition we have placed cross hairs in Figure 3 at onset latency $3.5 \mathrm{~ms}$ [the earliest onset of StimulusTA effects from the PMRF (Davidson and Buford, 2004) and PWHM is $9 \mathrm{~ms}$, used previously for SpikeTA effects of M1 neurons (Schieber and Rivlis, 2005)]. Most SpikeTA effects produced by PMRF neurons (69\%; 29 of 42) had onset latencies $>3.5$ ms. Most (66\%; 28 of 42) had a PWHM $<9.0 \mathrm{~ms}$. Slightly more than half of the PMRF SpikeTA effects $(55 \%, 23$ of 42) had both an onset latency $>3.5$ $\mathrm{ms}$ and a PWHM $<9.0 \mathrm{~ms}$, potentially representing pure postspike effects.

Onset latencies of PMRF neuron SpikeTA effects tended to be slightly earlier for suppression $(3.1 \pm 3.9 \mathrm{~ms})$ than facilitation $(6.7 \pm 4.1 \mathrm{~ms})(p<0.01, t$ test $)$. Suppression was more common among SpikeTA effects with onset latencies $<3.5 \mathrm{~ms}(62 \%, 8$ of $13)$, whereas facilitation was more common $(72 \%, 21$ of 29$)$ among SpikeTA effects with onset latencies $>3.5 \mathrm{~ms}(p<0.05$, Fisher's exact test). PWHM tended to be wider for suppression $(9.0 \pm 3.9 \mathrm{~ms})$ than for facilitation $(6.0 \pm 3.1 \mathrm{~ms})(p<0.05, t$ test). Although SpikeTA effects with PWHM $<9.0 \mathrm{~ms}$ were more frequently facilitative (18) than suppressive (9), and SpikeTA effects with a PWHM $>9.0$ were more frequently suppressive (10) than facilitative (5), this trend was not significant $\left(p>0.05, \chi^{2}\right.$ test). Overall, their earlier onsets and wider peak widths suggest that suppressive SpikeTA effects produced by PMRF neurons 


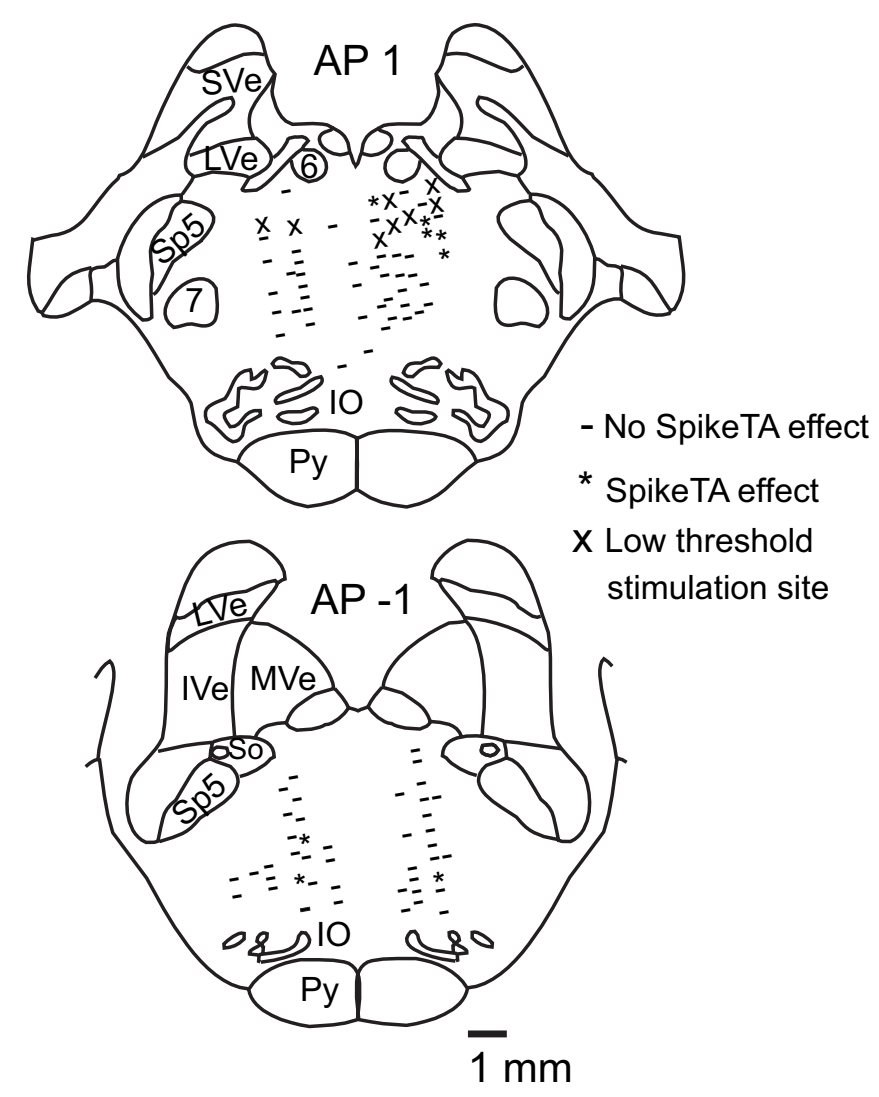

Figure 4. Anatomical reconstruction of recording sites for all monkeys at anterior-posterior coordinates - 1 and 1 (Szabo and Cowan, 1984). Asterisks indicate sites where neurons produced significant SpikeTA effects; dashes indicate sites without SpikeTA effects; $X$ indicates low-threshold stimulation sites for the StimulusTA plotted (monkeys I and G). Sve, Superior vestibular nucleus; LVe, lateral vestibular nucleus; MVe, medial vestibular nucleus; IVe, inferior vestibular nucleus; 6, abducens nucleus; 7, facial nucleus; 10 , inferior olive; Py, pyramidal tract; Sp5, spinal trigeminal nucleus; So, solitary tract.

were more likely to include synchrony, whereas facilitative SpikeTA effects were more likely to represent pure postspike effects.

SpikeTA effects of PMRF neurons were of relatively low amplitude. Facilitative effects had PPCs ranging from 1.7 to $13.7 \%$ (mean $\pm \mathrm{SD}, 4.6 \pm 2.9$ ) and MPCs ranging from 1.4 to $6.8 \%$ $(3.2 \pm 1.7)$. Suppressive effects had PPCs ranging from -1.3 to $-14.4 \%(-4.8 \pm 2.8)$ and MPCs ranging from -0.8 to $-10.3 \%$ $(-3.3 \pm 2.0)$. We found no systematic differences in these amplitude measures depending on whether the muscle was ipsilateral versus contralateral to the recorded neuron.

\section{PMRF neurons producing SpikeTA effects have similar spatial locations}

Rather than being scattered throughout the PMRF, neurons that produced SpikeTA effects appeared to be clustered in two small regions. Figure 4 presents the reconstructed location of eight PMRF neurons that produced SpikeTA effects (asterisks). To illustrate the entire territory sampled, sites in monkeys I and G at which PMRF neurons that produced no SpikeTA effect were recorded are plotted as well (Fig. 4, dashes). Ten of the PMRF neurons that produced SpikeTA effects were recorded within a restricted zone $\sim 2-4 \mathrm{~mm}$ ventral to the abducens nucleus and extending slightly rostrally [anteroposterior (AP), 1-2]. Recording sites in monkeys I and G were located throughout the PMRF, whereas recording sites in monkeys $\mathrm{C}$ and $\mathrm{D}$ were located more often in the region ventral to the abducens nucleus, where most SpikeTA effects were found. Consequently, a higher percentage of recorded PMRF neurons produced SpikeTA effects in monkeys C and D (7\%, 4 of 57$)$ than in monkeys I and $\mathrm{G}(3 \%, 10$ of 252$)$. Sites in monkeys I and $\mathrm{G}$ where single microstimulation pulses at $30 \mu \mathrm{A}$ were sufficient to evoke twitches in the UTr and shoulder muscles (Fig. 4, X) were found in this same rostrodorsal region of the PMRF (Davidson and Buford, 2006). The remaining five PMRF neurons that produced SpikeTA effects were recorded in a separate region $\sim 5-7 \mathrm{~mm}$ ventral and caudal (AP, 0 to -1 ) to the abducens nucleus. No systematic differences in the SpikeTA effects produced by rostrodorsal versus caudoventral PMRF neurons were observed.

\section{Discussion}

To our knowledge, this is the first report of SpikeTA effects produced bilaterally in arm and shoulder muscles from supraspinal neurons in the monkey. SpikeTA effects from PMRF neurons showed reciprocal organization, both between the flexors and extensors of a given limb and between homologous muscles in the ipsilateral and contralateral upper extremities. The most prevalent SpikeTA effect pattern produced by PMRF neurons included facilitation in ipsilateral flexors and contralateral extensors with simultaneous suppression in ipsilateral extensors and contralateral flexors.

These reciprocal patterns of PMRF output are consistent with patterns observed previously using electrical stimulation (Sprague and Chambers, 1954; Shapovalov et al., 1971; Drew and Rossignol, 1990; Davidson and Buford, 2004, 2006), suggesting that the output effects of single-pulse microstimulation in the PMRF result from stimulating PMRF neurons directly rather than fibers of passage. The similarity between SpikeTA and StimulusTA effects obtained at the same site further supports this conclusion. Although we recorded throughout much of the PMRF, most neurons that produced SpikeTA effects were found in a small region ventral to the abducens nucleus.

\section{Pure and synchrony SpikeTA effects from the PMRF}

Acute studies have demonstrated monosynaptic connections from reticulospinal neurons to spinal motoneurons of neck, back, hindlimb, and forelimb muscles in the cat (Peterson, 1979) and to lumbar motoneurons in the monkey (Shapovalov et al., 1971). Reticulospinal projections to cervical motoneurons have not been studied in monkeys, but many of the present PMRF SpikeTA effects had onset latency and PWHM characteristics consistent with monosynaptic connections to arm and shoulder motoneurons. Some PMRF neuron SpikeTA effects had early onsets and/or wide peaks, suggesting synchronization with other neurons that had inputs to the same motoneuron pool. Many corticospinal axons send collaterals to PMRF neurons (Keizer and Kuypers, 1989; Matsuyama and Drew, 1997; Kably and Drew, 1998) and are a potential source of such synchronization.

Synchrony was especially evident for suppression SpikeTA effects. The average onset latency for suppression $(3.1 \mathrm{~ms})$ was earlier than for facilitation $(6.7 \mathrm{~ms})$. Corticospinal neurons that project to spinal inhibitory interneurons and also send collaterals to reticulospinal neurons projecting to the same inhibitory interneurons could produce the early onset of synchrony suppression effects from PMRF neurons. Our observation that only 14 of 309 PMRF neurons produced SpikeTA effects further emphasizes that most reticulospinal neurons influence spinal motoneurons indirectly through interneurons. 


\section{Species comparisons}

Previous findings in the cat (Sprague and Chambers, 1954; Drew and Rossignol, 1990) and the monkey (Davidson and Buford, 2004, 2006), indicate that PMRF outputs to arm shoulder muscles are similar in these species. SpikeTA effects produced by cat PMRF neurons (Schepens and Drew, 2006), however, show four notable differences when compared with those observed in the monkey. First, PMRF neuron SpikeTA effects were more prevalent in the cat $(\sim 40 \% ; 21$ of 50 for left arm reach trials or 17 of 49 for right arm reach trials) than in the monkey ( $~ 5 \%, 14$ of 292$)$. In part, this difference may reflect the use of antidromic stimulation from the cat spinal cord to identify reticulospinal neurons. Additionally, this difference might reflect greater PMRF activation in cats reaching from a standing position than in monkeys reaching from a sitting position. Second, the vast majority of cat SpikeTA effects were in ipsilateral extensors and were suppressive $(82 \%)$. In the present monkeys, we found 15 (36\%) ipsilateral and $8(19 \%)$ contralateral facilitative effects, and $13(31 \%)$ ipsilateral and $6(14 \%)$ contralateral suppressive effects (45\%), which were reciprocally organized in ipsilateral and contralateral flexors and extensors. Third, SpikeTA effects in the cat were not reported to have early onsets or wide peaks (or troughs) suggestive of synchrony, whereas 13 of the 42 effects in the present monkeys did. Fourth, neurons producing SpikeTA effects showed no regional localization within the feline PMRF, whereas in the present monkeys such cells were located mainly in the caudal NRPc and rostral NRGc, just ventral to the abducens nucleus. Finally, we note that, in the cat, PMRF neurons produced SpikeTA effects in hindlimb and axial muscles as well as forelimb muscles; only forelimb muscles were examined in the monkey. Future experiments will determine whether these differences can be attributed to differences in the behavioral tasks and experimental techniques, or to physiological differences between species. Nevertheless, during reaching in both species, certain PMRF neurons produce short-latency, spike-locked effects in arm and shoulder muscles.

\section{Conclusions}

We found that certain PMRF neurons produce bilateral SpikeTA effects with a double reciprocal output organization, facilitating ipsilateral flexors and contralateral extensors while suppressing ipsilateral extensors and contralateral flexors. This general pattern of output effects would support concurrent flexion of the ipsilateral extremity and extension of the contralateral extremity, a pattern that likely developed in quadrupeds to help coordinate the forelimbs during locomotion or for postural adjustments (Drew and Rossignol, 1984). The same pattern could support reaching from a quadrupedal stance, in which additional weight is born on the extended forelimb as the other forelimb is lifted to reach (Schepens and Drew, 2006). In primates reaching from a seated position, we speculate that the premotor and motor cortices (Keizer and Kuypers, 1989; Matsuyama and Drew, 1997) also recruit PMRF neurons to coordinate postural adjustments between the forelimbs during reaching movements.

\section{References}

Buford JA, Davidson AG (2004) Movement-related and preparatory activity in the reticulospinal system of the monkey. Exp Brain Res 159:284-300.

Davidson AG, Buford JA (2004) Motor outputs from the primate reticular formation to shoulder muscles as revealed by stimulus triggered averaging. J Neurophysiol 92:83-95.

Davidson AG, Buford JA (2006) Bilateral actions of the reticulospinal tract on arm and shoulder muscles in the monkey: stimulus triggered averaging. Exp Brain Res 173:25-39.

Drew T, Rossignol S (1984) Phase-dependent responses evoked in limb muscles by stimulation of medullary reticular formation during locomotion in thalamic cats. J Neurophysiol 52:653-675.

Drew T, Rossignol S (1990) Functional organization within the medullary reticular formation of intact unanesthetized cat. I. Movements evoked by microstimulation. J Neurophysiol 64:767-781.

Jankowska E, Hammar I, Slawinska U, Maleszak K, Edgley SA (2003) Neuronal basis of crossed actions from the reticular formation on feline hindlimb motoneurons. J Neurosci 23:1867-1878.

Kably B, Drew T (1998) Corticoreticular pathways in the cat. II. Discharge activity of neurons in area 4 during voluntary gait modifications. J Neurophysiol 80:406-424.

Keizer K, Kuypers HG (1989) Distribution of corticospinal neurons with collaterals to the lower brain stem reticular formation in monkey (Macaca fascicularis). Exp Brain Res 74:311-318.

Matsuyama K, Drew T (1997) Organization of the projections from the pericruciate cortex to the pontomedullary brainstem of the cat: a study using the anterograde tracer Phaseolus vulgaris-leucoagglutinin. J Comp Neurol 389:617-641.

McKiernan BJ, Marcario JK, Karrer JH, Cheney PD (1998) Corticomotoneuronal postspike effects in shoulder, elbow, wrist, digit, and intrinsic hand muscles during a reach and prehension task. J Neurophysiol 80:1961-1980.

Peterson BW (1979) Reticulospinal projections to spinal motor nuclei. Annu Rev Physiol 41:127-140.

Poliakov AV, Schieber MH (1998) Multiple fragment statistical analysis of post-spike effects in spike-triggered averages of rectified EMG. J Neurosci Methods 79:143-150.

Schepens B, Drew T (2006) Descending signals from the pontomedullary reticular formation are bilateral, asymmetric, and gated during reaching movements in the cat. J Neurophysiol 96:2229-2252.

Schieber MH, Rivlis G (2005) A spectrum from pure post-spike effects to synchrony effects in spike-triggered averages of electromyographic activity during skilled finger movements. J Neurophysiol 94:3325-3341.

Shapovalov AI, Tamarova ZA, Karamian OA, Kurchavyi GG (1971) [Reticulospinal and vestibulospinal synaptic effects on monkey lumbar motor neurons]. Neirofiziologiia 3:408-417.

Sprague JM, Chambers WW (1954) Control of posture by reticular formation and cerebellum in the intact, anesthetized and unanesthetized and in the decerebrated cat. Am J Physiol 176:52-64.

Szabo J, Cowan WM (1984) A sterotaxic atlas of the brain of the cynomolgus monkey (Macaca fascicularis). J Comp Neurol 222:265-300. 